\title{
Surface topography and water contact angle of sandblasted and thermally annealed glass surfaces
}

Cite as: J. Chem. Phys. 150, 054701 (2019); https://doi.org/10.1063/1.5080200

Submitted: 06 November 2018 . Accepted: 10 January 2019. Published Online: 04 February 2019

B. N. J. Persson
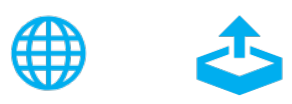

\section{ARTICLES YOU MAY BE INTERESTED IN}

Ice friction: Glacier sliding on hard randomly rough bed surface

The Journal of Chemical Physics 149, 234701 (2018); https://doi.org/10.1063/1.5055934

Thermodynamic picture of vitrification of water through complex specific heat and entropy: A journey through "no man's land"

The Journal of Chemical Physics 150, 054502 (2019); https://doi.org/10.1063/1.5079594

High-temperature superconductivity in alkaline and rare earth polyhydrides at high pressure: A theoretical perspective

The Journal of Chemical Physics 150, 050901 (2019); https://doi.org/10.1063/1.5079225
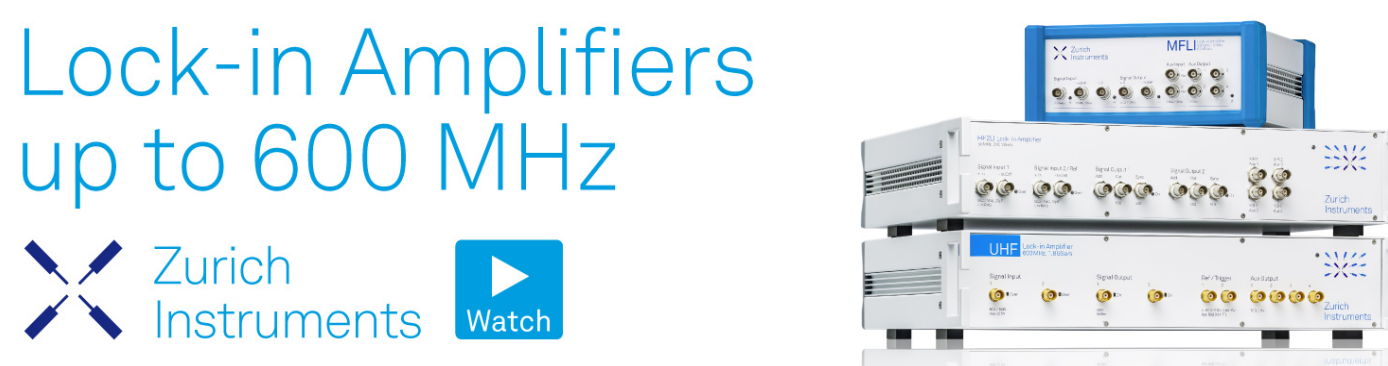


\title{
Surface topography and water contact angle of sandblasted and thermally annealed glass surfaces
}

\author{
Cite as: J. Chem. Phys. 150, 054701 (2019); doi: 10.1063/1.5080200 \\ Submitted: 6 November 2018 - Accepted: 10 January 2019 • \\ Published Online: 4 February 2019
}

B. N. J. Persson

\section{AFFILIATIONS}

PGI-7, FZ Jülich, Germany and Multiscale Consulting, Wolfshovener Str 2, 52428 Jülich, Germany

\begin{abstract}
Surface roughness has a huge influence on most tribology properties. Sandblasting is a standard way to produce surface roughness in a controlled and reproducible way. Sometimes the sandblasted surfaces are annealed to reduce the roughness and reduce the sharpness of the roughness. We study the nature of the surface roughness of sandblasted silica glass surfaces and how it is modified by annealing at different temperatures. The surface roughness decreases with increasing annealing temperature due to viscous flow of the glass driven by the surface tension. However, the skewness and kurtosis remain nearly unchanged. Optical pictures of the annealed glass surfaces exhibit cell-like structures (cell diameter $\approx 20-40 \mu \mathrm{m}$ ), which we interpret as micro-cracks. The concentration of micro-cracks increases with increasing annealing temperature. The micro-cracks result in a (advancing) water contact angle which decreases with increasing annealing temperature, which is opposite to what is expected from the theory if no micro-cracks would occur.
\end{abstract}

Published under license by AIP Publishing. https://doi.org/1 0.1063/1.5080200

\section{INTRODUCTION}

Surface roughness has a big influence on many physical phenomena such as sliding friction, adhesion, contact mechanics, contact stiffness, fluid flow at interfaces, and the electric and thermal contact resistance. ${ }^{1-8}$ It is therefore important to understand how different surface preparation methods influence the surface topography. Here I study how thermal annealing reduces and modifies the surface roughness of sandblasted glass surfaces. I note that thermal annealing is a standard way to reduce the surface roughness of glassy materials, and understanding how this process influences the surface topography is of some interest. The influence of thermal annealing on the topography of ion-bombarded surfaces was studied by Krim et al. in Ref. 9 .

Experiments and model calculations ${ }^{9-12}$ have shown that many surfaces of solids have self-affine fractal properties with the Hurst exponent $0<\mathrm{H}<1$ but typically ${ }^{12} 0.7<\mathrm{H}<1$. Sandblasted surfaces also have fractal properties with a Hurst exponent $H \approx 1$ (and the fractal dimension $D_{\mathrm{f}}=3-H \approx 2$ ). This implies that when a region of such a surface is magnified it appears the same as before the magnification. Sandblasted surfaces always have negative skewness and are therefore not randomly rough surfaces. ${ }^{13}$ The origin of the negative skewness can be explained as follows (see Fig. 1): The particles which impact a surface during sandblasting tend to reduce the height of the high asperities, in particular, when they impact the surface at a non-zero angle (see Ref. 12). On the other hand, deep cavities are left nearly unchanged. This will result in negative skewness.

When a sandblasted surface is heat treated (annealed), the surface will get smoothed. The driving force for this is the reduction in the surface area and hence in the surface energy. The speed of smoothing depends on the viscosity $\eta$ of the glassy material (which depends on the temperature) and on the glass surface free energy per unit surface area $\gamma$ (also denoted as the surface tension). As will be shown below, there appears to be no systematic change in the skewness and kurtosis with increasing annealing temperature.

\section{EXPERIMENTAL RESULTS AND DISCUSSION}

We have sandblasted 7 rectangular borosilicate glass plates $(3 \mathrm{~cm} \times 3 \mathrm{~cm} \times 0.5 \mathrm{~cm})$ and annealed 6 of them for $1 \mathrm{~h}$ 


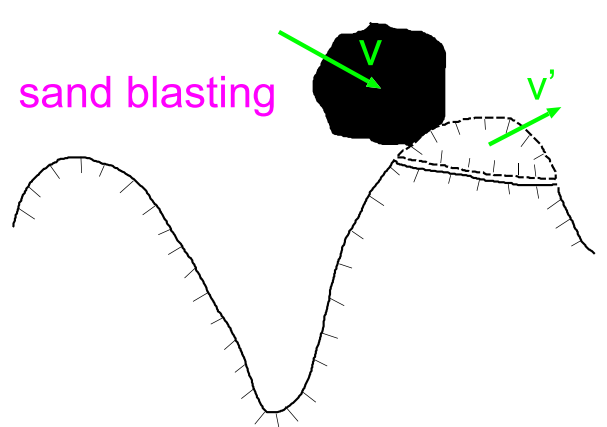

FIG. 1. Sandblasting particles tend to reduce the height of high asperities, in particular, when they impact the surface at a non-zero angle (see Ref. 12), while deep cavities are left nearly unchanged. Sandblasted solid surfaces therefore always have negative skewness.

at different temperatures from $\mathrm{T}=750{ }^{\circ} \mathrm{C}$ to $\mathrm{T}=860{ }^{\circ} \mathrm{C}$. The annealing cycle consisted in putting a glass plate in the cold oven, which then was heated to the final temperature in about $2 \mathrm{~h}$, and then kept at this temperature for $1 \mathrm{~h}$ and then cooled down to $200{ }^{\circ} \mathrm{C}$ in about $10 \mathrm{~h}$.

We have studied the surface topography of all the surfaces using Mitutoyo Portable Surface Roughness Measurement Surftest SJ-410 with a diamond tip with the radius of curvature $R=2 \mu \mathrm{m}$ and with the tip-substrate repulsive force $F_{N}=0.75 \mathrm{mN}$. The scan length was $L=20 \mathrm{~mm}$, and the tip speed was $v=50 \mu \mathrm{m} / \mathrm{s}$. From the line scans $z=h(x)$, we calculate the one-dimensional (1D) surface roughness power spectra of the surfaces. If we choose the $z=0$ plane so that $\langle h\rangle$ $=0$, the $1 \mathrm{D}$ power spectrum is given by ${ }^{12,14-16}$

$$
\mathrm{C}_{1 \mathrm{D}}(q)=\frac{1}{2 \pi} \int d x\langle h(x) h(0)\rangle e^{-i q x},
$$

where $\langle\ldots\rangle$ stands for ensemble averaging. The surface meansquare roughness, skewness, and kurtosis are defined as

$$
\left\langle h^{2}\right\rangle, \quad \frac{\left\langle h^{3}\right\rangle}{\left\langle h^{2}\right\rangle^{3 / 2}}, \quad \text { and } \quad \frac{\left\langle h^{4}\right\rangle}{\left\langle h^{2}\right\rangle^{2}},
$$

respectively. A randomly rough surface has a Gaussian height distribution and hence zero skewness and the kurtosis equal to 3 .

We also present optical pictures of two of the surfaces and water contact angles, which depend on the surface topography, from all the surfaces.

At room temperature, silica glass behaves as an elastically stiff and brittle material. However, at high temperature, it behaves as a viscous fluid. The viscosity of borosilicate glass changes from ${ }^{17} \eta \approx 3 \times 10^{7} \mathrm{~Pa} \mathrm{~s}$ at $\mathrm{T}=750{ }^{\circ} \mathrm{C}$ to $\approx 1 \times 10^{6} \mathrm{~Pa} \mathrm{~s}$ at $\mathrm{T}=840^{\circ} \mathrm{C}$. The surface free energy per unit surface area (the surface tension) in the normal atmosphere in the same temperature range is ${ }^{18} \gamma \approx 0.3 \mathrm{~J} / \mathrm{m}^{2}$.

\section{A. Surface topography}

Figure 2 shows the surface height coordinate over $1 \mathrm{~mm}$ long tracks for the sandblasted but not-annealed glass surface

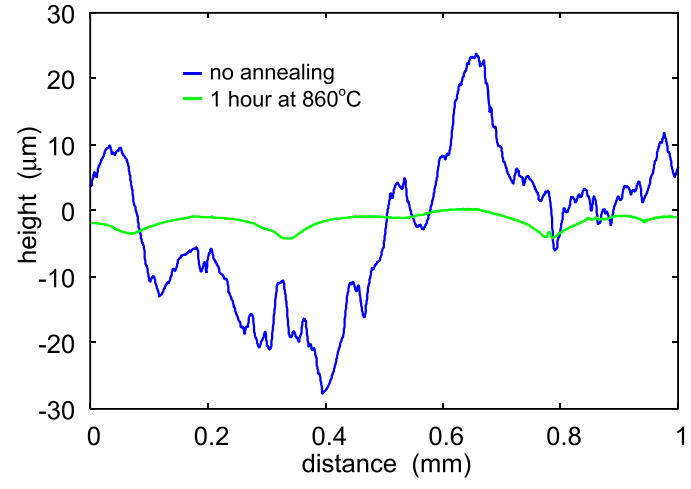

FIG. 2. The surface height coordinate over $1 \mathrm{~mm}$ long tracks for the sandblasted but not-annealed glass surface (blue) and for the glass surfaces annealed at $860^{\circ} \mathrm{C}$ (green).

(blue) and for the glass surfaces annealed at $860{ }^{\circ} \mathrm{C}$ (green). Note the strong reduction (by a factor of $\approx 10$ ) in the surface roughness for the annealed surface. Note also the peculiar form of the height profile of the annealed surface, with asperities with a large radius of curvature separated by valleys with much smaller radius of curvature. It is clear that this surface has negative skewness. This can also be seen in Fig. 3 which shows the height probability distribution of a sandblasted silica glass surface before annealing (blue) and after annealing for $1 \mathrm{~h}$ at $\mathrm{T}=860{ }^{\circ} \mathrm{C}$ (green). Note that both the surfaces have negative skewness. Negative skewness has been observed for other sandblasted surfaces, e.g., PMMA (glassy polymer). ${ }^{19}$

The variation of the skewness, kurtosis, and the rmsroughness with the annealing temperature is shown in Fig. 4. While the rms-roughness tends to decrease with increasing annealing temperature, there is no definitive dependency

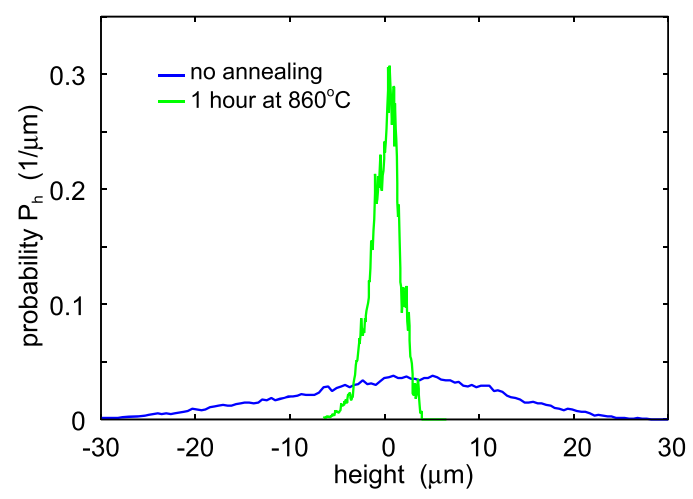

FIG. 3. The height probability distribution of a sandblasted silica glass surface before annealing (blue) and after annealing for $1 \mathrm{~h}$ at $T=860^{\circ} \mathrm{C}$ (green). Note that both the surfaces have negative skewness. Sandblasted surfaces always have negative skewness because the sandblasting particles tend to reduce the height of high asperities, in particular, when they impact the surface at a non-zero angle (see Ref. 12). 


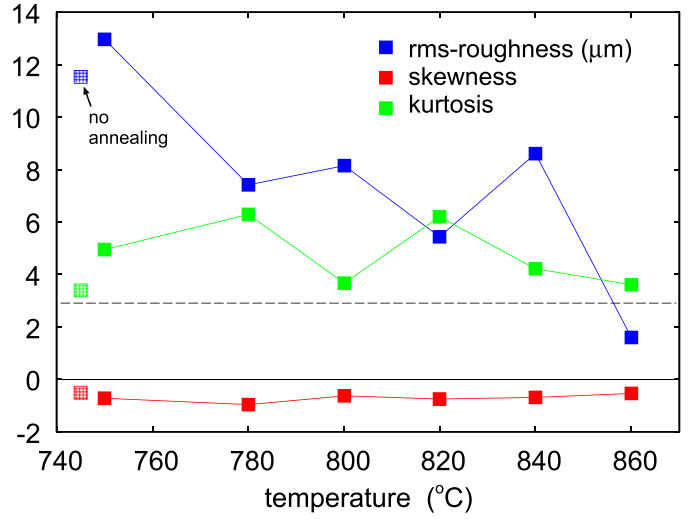

FIG. 4. The root-mean-square roughness, the skewness, and the kurtosis as a function of the annealing temperature ( $1 \mathrm{~h}$ annealing time)

of the skewness and the kurtosis on the annealing temperature. The non-monotonous behavior of the measured data as a function of the annealing temperature could reflect fluctuations in the sandblasting conditions of different glass plates; sandblasting was performed "by hand" under nominally identical conditions, but some variations in the sandblasting conditions cannot be avoided. Unfortunately, the surface topography of different plates was not measured before the thermal annealing.

\section{B. Surface roughness power spectra}

Figure 5 shows the 1D-surface roughness power spectra of the sandblasted glass surfaces before annealing (blue curve) and after annealing for $1 \mathrm{~h}$ at $750{ }^{\circ} \mathrm{C}$ (black) and at $840{ }^{\circ} \mathrm{C}$ (red). Note that after annealing at $750{ }^{\circ} \mathrm{C}$ the short wavelength roughness is reduced but the long wavelength roughness is nearly unchanged. This is due to the influence of the glass surface tension $\gamma$ on the roughness profile. Thus, the viscous glass will flow in such a way as to reduce the surface area and the

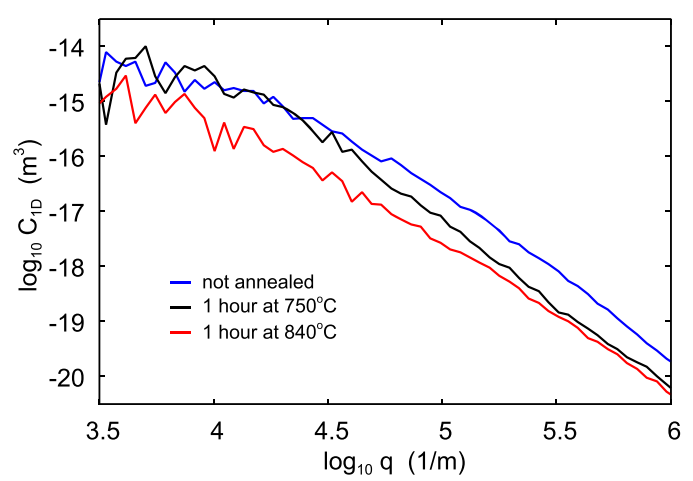

FIG. 5. The 1D surface roughness power spectra of the not-annealed sandblasted glass surfaces (blue curve) and the glass surfaces annealed for $1 \mathrm{~h}$ at $750^{\circ} \mathrm{C}$ (black) and at $840^{\circ} \mathrm{C}$ (red). surface energy. From dimensional argument, we expect that a surface roughness component with wavenumber $q=2 \pi / \lambda$ (where $\lambda$ is the wavelength) will get smoothed on the time scale $\tau_{q} \approx \eta /(q \gamma)$; this is the only way to construct a quantity with the unit of time from the quantities characterizing the problem (see also Appendix A). Here we have neglected the influence of gravity which is a good approximation as long as the capillary length ${ }^{20} \lambda_{\mathrm{c}}=(\gamma / \rho g)^{1 / 2}$ is longer than the longest wavelength roughness component. In the present case, $\lambda_{\mathrm{c}} \approx 1 \mathrm{~cm}$ so gravity can be neglected.

For the glass at $\mathrm{T}=750^{\circ} \mathrm{C}$, we have ${ }^{18} \gamma \approx 0.3 \mathrm{~J} / \mathrm{m}^{2}$ and the viscosity $\eta \approx 3 \times 10^{7}$ Pa s. Hence during $1 \mathrm{~h}(\tau=3600 \mathrm{~s})$ annealing time the surface tension will smooth the roughness components with wavenumber $q>\eta /(\tau \gamma) \approx 3 \times 10^{4} \mathrm{~m}^{-1}$ in good agreement with Fig. 5. Since the smoothing is strongest for the shorter wavelength roughness, the slope of the $C(q)$ curve (on the log-log scale) increases, corresponding to an apparent increase in the Hurst exponent. ${ }^{10,11}$ For $\mathrm{T}=840{ }^{\circ} \mathrm{C}$, we have $\eta \approx 1 \times 10^{6}$ Pa s giving $\eta /(\tau \gamma) \approx 1 \times 10^{3} \mathrm{~m}^{-1}$. This is again consistent with Fig. 5 which shows that for this case smoothing has extended to the longest length-scale probed.

Figure 6 shows the 1D surface roughness power spectra of all the surfaces annealed at different temperatures. Note the strong drop in the magnitude of the power spectrum when the annealing temperature increases from $840^{\circ} \mathrm{C}$ to $860^{\circ} \mathrm{C}$. Thus, annealing at $860^{\circ} \mathrm{C}$ results in a surface with a rms roughness $\sim 10$ times smaller than for the not-annealed surface.

\section{Optical pictures of the glass surfaces}

After the heat treatment (annealing), the sandblasted glass surface appears more "shiny" to the naked eye. Clearly, most light is diffusely scattered from the sandblasted surface, but for the annealed glass surfaces, some fraction of the incident light is nearly specular reflected. This can also be seen in optical pictures of the surfaces. Thus, Fig. 7 shows optical pictures of the sandblasted but not-annealed surface (a) and after annealing at $860^{\circ} \mathrm{C}$ for $1 \mathrm{~h}$ (b). Note the light reflections from the annealed surface from the 8 light diodes used to illuminate

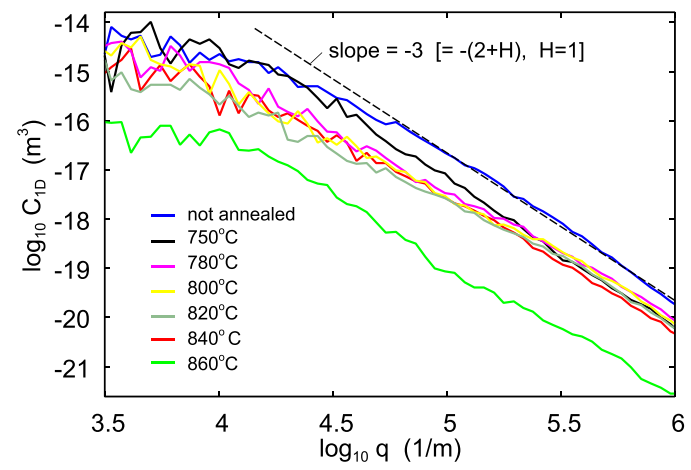

FIG. 6. The 1D surface roughness power spectra of sandblasted glass surfaces annealed for $1 \mathrm{~h}$ at different temperatures. Note the strong drop in the magnitude of the power spectrum when the annealing temperature increases from $840{ }^{\circ} \mathrm{C}$ to $860^{\circ} \mathrm{C}$. 
(a)

not annealed

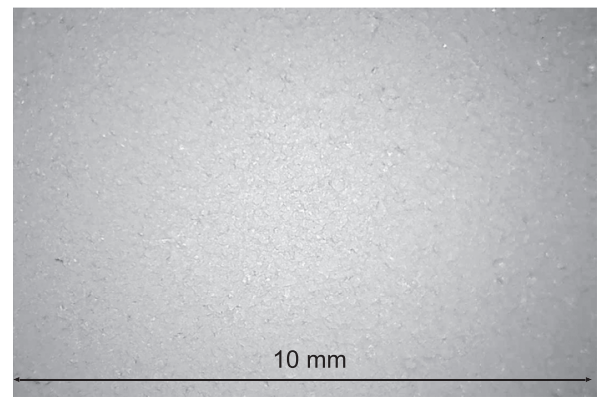

(b) annealed $860^{\circ} \mathrm{C}$ for 1 hour

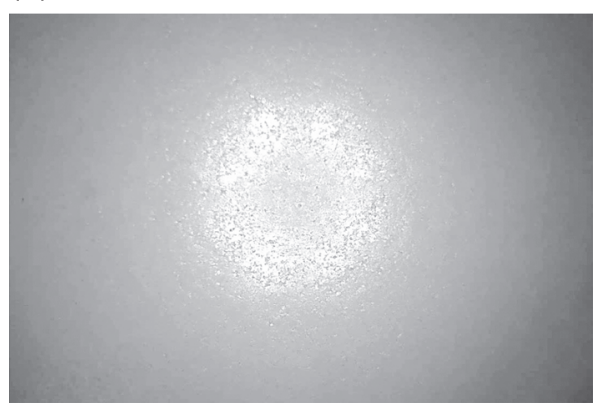

FIG. 7. Optical pictures of the sandblasted and not-annealed surface (a) and after annealing at $860^{\circ} \mathrm{C}$ for $1 \mathrm{~h}(\mathrm{~b})$. Note the light reflections in the annealed surface from the 8 light diodes used to illuminate the surface. For the not-annealed surface, the light scatters diffusely.

the surface. For the not-annealed surface, the light is diffusely scattered.

Figures 8 and 9 show optical pictures of the same surfaces as in Fig. 7 but at higher magnification. Note that while the not-annealed surface appears randomly rough, the annealed

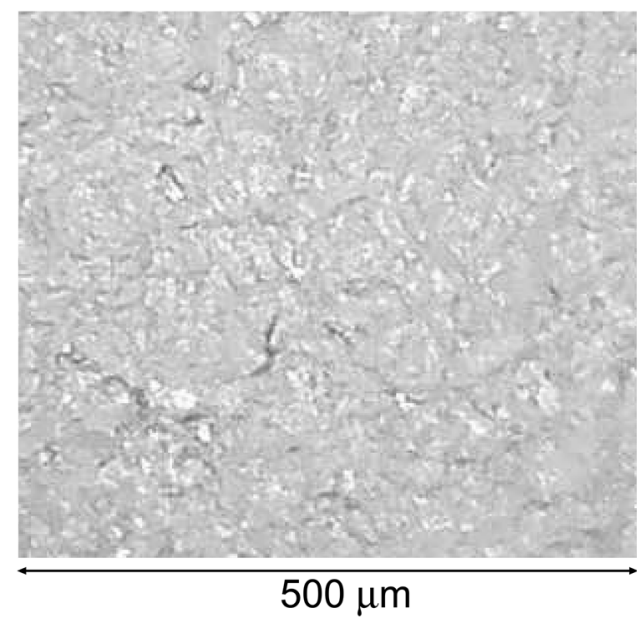

FIG. 8. Optical picture of the sandblasted and not-annealed surface at higher magnification.

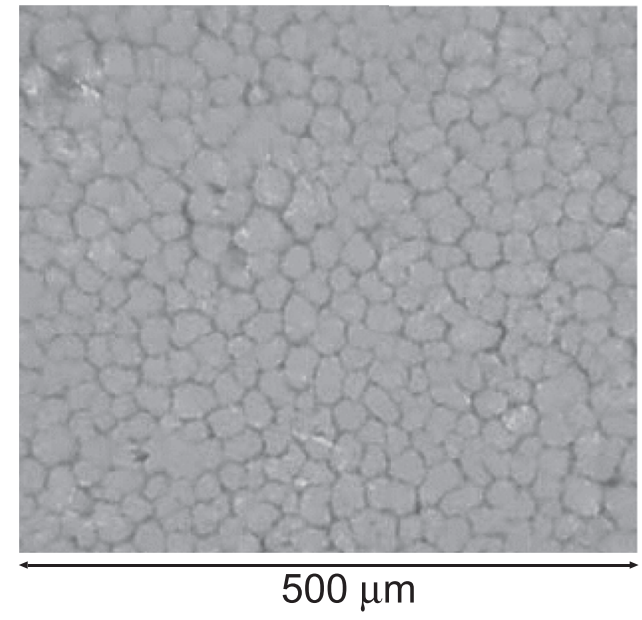

FIG. 9. Optical picture of the sandblasted glass surface after annealing at $860^{\circ} \mathrm{C}$ for $1 \mathrm{~h}$. Note the cell-like structure of the surface.

surface has developed a cell-like structure, where the cell diameter is of order $\sim 20-40 \mu \mathrm{m}$. Optical pictures of the glass surfaces annealed at the lower temperatures show a lower concentration of the defects (see Fig. 10). The defects could be due to the formation of small crystalline regions or due to a distribution of crack-like defects, e.g., formed during the cooling process. In the stylus topography measurements (and the power spectra), we do not observe any structures with the periodicity in the range $\sim 20-40 \mu \mathrm{m}$ so it appears as if the cell-like structures are not associated with any height fluctuations of the glass surface. This is expected if the defects are micro-cracks. Thus, if the surface separation between the walls in the cracks is smaller than the radius of curvature of the diamond tip (i.e., less than a few micrometer), then the

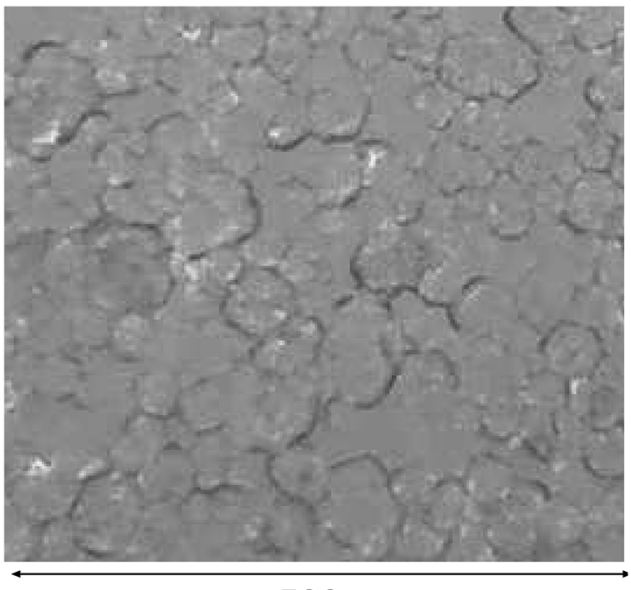

$500 \mu \mathrm{m}$

FIG. 10. Optical picture of the sandblasted glass surface after annealing at $750{ }^{\circ} \mathrm{C}$ for $1 \mathrm{~h}$. Note the much lower concentration of crack-like defects on this surface compared to the case of annealing at $860^{\circ} \mathrm{C}$ (see Fig. 9). 
cracks cannot be observed in the stylus topography. When flat (not sandblasted) glass surfaces are exposed to the same heat cycles as above, crack-like defects form on the surfaces with a similar appearance as on the sandblasted surfaces. In general, while sandblasted surfaces become smoother after the heat treatment, smooth glass surfaces become rougher (see Appendix B).

We note that the hexagonal (or polygonal) pattern of vertical cracks has been observed for many other systems which have undergone (fast enough) cooling, such as lava (see Appendix C).

\section{Water contact angle}

A perfectly clean silica glass is wet by water, i.e., the water contact angle is zero. However glass surfaces exposed to the normal atmosphere quickly get contaminated by organic molecules, some of which bind with strong covalent bonds to the glass surface. These molecules cannot be removed with any standard cleaning fluids such as acetone or isopropanol. Thus, the (advancing) water contact angle of glass surfaces exposed to the normal atmosphere, even if cleaned with organic fluids, shows non-zero contact angles. ${ }^{22}$

We have measured the water contact angle on all the sandblasted and annealed glass surfaces (see Fig. 11 for one example). The surfaces were cleaned with acetone and isopropanol in order to remove weakly bound organic contamination. Figure 12 shows the water advancing contact angle on smooth clean borosilicate glass (blue squares), on sandblasted glass (green squares), and on sandblasted glass which was annealed at different temperatures for $1 \mathrm{~h}$ (red squares). Note that on the annealed samples the contact angle tends to decrease with increasing annealing temperature. This is at first somewhat unexpected as one expect from theory that the contact angle increases as the surface rmsslope decreases. ${ }^{20,21}$ However, if one assumes that the celllike structures observed on the annealed surfaces are micro cracks, water can penetrate into the cracks and lower the total energy and the contact angle. Since the density of the cracklike defects increases with increasing annealing temperature (compare Figs. 9 and 8), it would explain the decrease in the contact angle with the increasing annealing temperature.

On the flat glass surface, the advancing water contact angle was $\theta \approx 45^{\circ}$, as is typical for contaminated

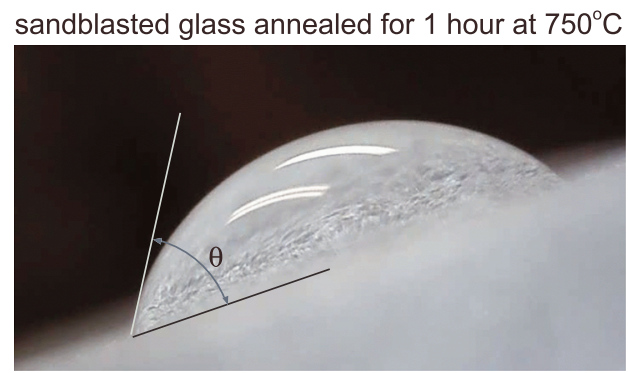

FIG. 11. A water droplet on the sandblasted borosilicate glass surface annealed for $1 \mathrm{~h}$ at $750^{\circ} \mathrm{C}$. The contact angle $\theta \approx 45^{\circ}$.

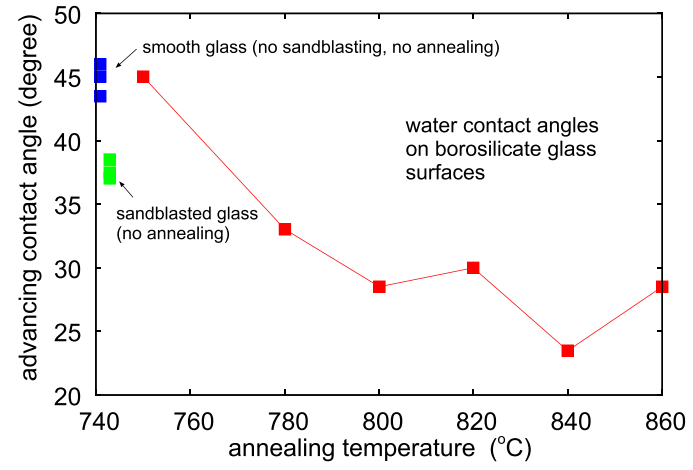

FIG. 12. Water advancing contact angle on smooth clean borosilicate glass (blue squares), on sandblasted glass (green squares), and on sandblasted glass which was annealed at different temperatures for $1 \mathrm{~h}$ (red squares).

borosilicate glass surfaces. ${ }^{22}$ On the sandblasted surface (but not-annealed), the contact angle was $\approx 38^{\circ}$. This is consistent with the theory prediction that if the contact angle on the flat surface is below $90^{\circ}$ then the contact angle for the rough surface (of the same material) is smaller than for the flat surface. ${ }^{20,21}$ When the sandblasted glass surface is annealed at $\mathrm{T}=750{ }^{\circ} \mathrm{C}$, the contact angle increases as compared to the sandblasted but not-annealed surface. This is again expected because of the reduction in the rms slope due to the annealing. Here we note that annealing at $\mathrm{T}=750{ }^{\circ} \mathrm{C}$ results in much less crack-like defects than for the surface annealed at the highest temperature, so the main effect is just the smoothing of the surface due to viscous flow of the glass.

\section{SUMMARY AND CONCLUSION}

We have studied the nature of the surface roughness of sandblasted silica glass surfaces, and how it is modified by annealing at different temperatures. The surface roughness decreases with increasing annealing temperature due to viscous flow of the glass driven by the glass surface tension. As expected from theory, we find that annealing at low temperature only reduce the short-wavelength roughness, say for $q$ $>q^{*}$, where the transition wavenumber $q^{*}$ moves to smaller wavenumber as the annealing temperature (or annealing time) increases. It appears as if annealing of the sandblasted surface has only a small influence on the surface skewness and kurtosis.

Optical pictures of the annealed surfaces show cell-like structures, which we interpret as micro-cracks. As the annealing temperature increase the concentration of micro-cracks increases.

The water advancing contact angle was measured on all the surfaces. For the sandblasted surfaces, it was found that the contact angle decreases with increasing annealing time. We propose that this surprising result is due to the increase in the concentration of the crack-like defects with increasing annealing temperature; water can penetrate into the cracks and lower the total energy and the contact angle. 


\section{ACKNOWLEDGMENTS}

I thank the glass workshop at FZ Jülich for preparing the glass samples. I thank Julia Persson (Multiscale Consulting) for performing the topography measurements. I thank G. Carbone for useful discussions. I thank DFG for the project support under the reference German Research Foundation DFG-Grant No. MU 1225/36-1. I also acknowledge the support by the DFG-Grant No. PE 807/12-1.

\section{APPENDIX A: SURFACE SMOOTHING}

The smoothing of the surface roughness of the silica glass by viscous flow can be described by the theory of capillary waves. According to this theory the relation between the wavenumber $q$ and frequency $\omega$ is s $^{23,24}$

$$
\gamma \rho q^{3}-\rho^{2} \omega^{2}+4 i \omega q^{2} \eta \rho+4 \eta^{2} q^{4}\left[1-\left(1+\frac{i \omega \rho}{\eta q^{2}}\right)^{1 / 2}\right]=0 .
$$

In the present case, $\omega \rho /\left(\eta q^{2}\right) \ll 1$ so that

$$
\left(1+\frac{i \omega \rho}{\eta q^{2}}\right)^{1 / 2} \approx 1+\frac{i \omega \rho}{2 \eta q^{2}}
$$

Substituting this in (A1) and using that $\rho \omega^{2} /\left(\gamma q^{2}\right) \ll 1$, we get after some simplifications

$$
\omega \approx-i \frac{q \gamma}{2 \eta} .
$$

Hence the surface roughness component

$$
h(x, t) \sim e^{i(q x-\omega t)} \sim e^{i q x-t / \tau_{q}^{\prime}}
$$

is damped in time with the relaxation time $\tau_{q}^{\prime}=2 \eta /(q \gamma)$. Thus

$$
h(q, t)=h(q, 0) e^{-t / \tau_{q}^{\prime}}
$$

and the power spectrum $C(q, t) \sim|h(q, t)|^{2}$ will decrease with time according to

$$
C(q, t)=C(q, 0) e^{-t / \tau_{q}},
$$

where $\tau_{q}=\eta /(q \gamma)$. This equation describes very well the onset of smoothing (with the increasing temperature) but not the smoothing for high temperature. In fact, the magnitude of the smoothing for large wavenumber in Fig. 6 hardly change when the annealing temperature increases from $750{ }^{\circ} \mathrm{C}$ to $840{ }^{\circ} \mathrm{C}$ where the glass viscosity (and hence $\tau_{q}$ ) changes with a factor of $\sim 30$. We note that the theory of capillary waves is based on the small-amplitude assumption, but we believe this is not the (main) origin of the deviation between the theory and the experiments. Modification of the surface during the cooling process, or plastic deformations or cracks caused by the sandblasting, which require higher annealing temperatures (or longer annealing time) to be removed, could also affect the power spectra.

\section{APPENDIX B: ANNEALED SMOOTH GLASS}

Here we present the power spectra for smooth glass plates which have been exposed to the same temperature

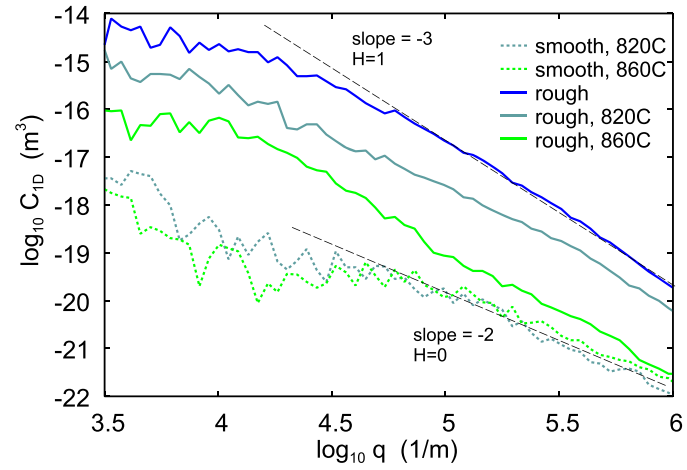

FIG. 13. The $1 \mathrm{D}$ surface roughness power spectra of the not-annealed sandblasted glass surfaces (blue curve) and the glass surfaces annealed for $1 \mathrm{~h}$ at $820^{\circ} \mathrm{C}$ (aquamarine) and at $860^{\circ} \mathrm{C}$ (green). The dotted lines show the power spectrum of smooth glass plates annealed for $1 \mathrm{~h}$ at $820^{\circ} \mathrm{C}$ (aquamarine) and at $860^{\circ} \mathrm{C}$ (green). The upper and lower dashed lines have the slope -3 and -2 corresponding to self-affine fractal surfaces with the Hurst exponent $H=1$ and $H=0$. The latter case (with the fractal dimension $D_{f}=3-H=3$ ) is the result expected for surfaces with frozen capillary waves, but the amplitude of the roughness in the present case is much too large to correspond to frozen thermally excited capillary waves.

cycles as for the sandblasted samples. The dotted lines in Fig. 13 show the power spectra when glass plates with smooth surfaces have been heat treated at $820{ }^{\circ} \mathrm{C}$ (aquamarine) and $860{ }^{\circ} \mathrm{C}$ (green). As expected, the surface roughness of these surfaces is much smaller than for the corresponding heat treated sandblasted surfaces, but still larger than for the smooth not heat-treated glass surface (not shown).

Optical pictures of the heat-treated smooth glass surfaces show crack-like defects similar to, but at a lower concentration, those of the heat treated sandblasted surfaces (see Fig. 16).

To summarize, for the smooth glass surface the heat treatment results in increased surface roughness. Thermal roughening of glass surfaces occurs when a temperature differential is created within the glass. As a cooled area contracts, compressible and tensile stresses develop potentially leading to plastic deformation and cracking of the glass during the cooling process. Thus, for example, if the surface of the glass is cooling faster than the interior, the non-uniform thermal contraction could result in the buildup of tensile stress at the surface which could be relieved by formation of surface cracks.

\section{APPENDIX C: CRACK PATTERN}

It has been shown ${ }^{25}$ (see also Refs. 26-29) that a hexagonal pattern of vertical cracks at a solid surface under thermal contraction has a lower free energy [elastic plus surface (or fracture) energy] than any other crack pattern. This is related to the fact that for any regular arrays with an equal column cross-section area, the hexagonal array has the smallest total column surface (or crack face) area. Hence a hexagonal (or more generally, polygonal) crack pattern is expected and also 


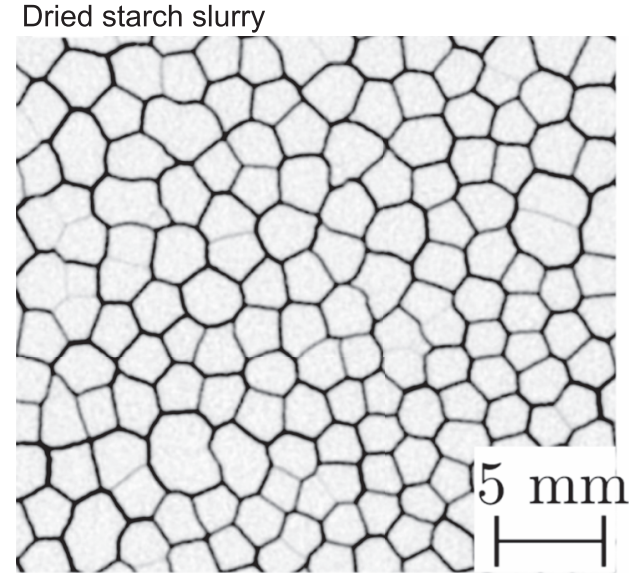

FIG. 14. Shrinkage crack pattern of dried starch slurry. Adapted from Ref. 26.

found for many systems such as the shrinkage cracks that develop during the cooling of solidified lava. The same preference for hexagonal columns has been found with drying starch slurry $^{26}$ and mud and thawing of permafrost soil. ${ }^{28}$ An example is shown in Fig. 14 from dried starch slurry which appears very similar to the pattern observed on the annealed glass surface (see Fig. 9).

The theoretical prediction of the diameter of the hexagonal unit on the annealed glass surface is a complex and not fully solved problem. ${ }^{26-29}$ It depends on the exact timedependent temperature profile at the glass surface which often is not accurately known.

It is well known that a (infinitely long) vertical crack of height $d$ in a semi-infinite solid reduces the elastic energy to (nearly) zero in a region which extends a distance $\approx d$ away

(a)

\section{non-interacting cracks}

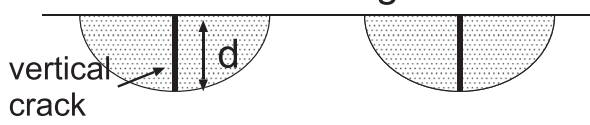

(b)

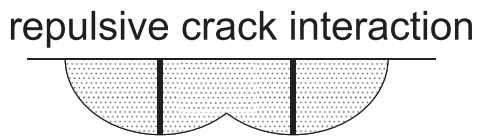

FIG. 15. (a) Two vertical cracks extending a distance $d$ into a semi-infinite elastic solid. The cracks reduce the elastic deformation energy (which is assumed to result from thermal contraction in a layer at the surface of the solid) to nearly zero in a region extending a distance $\approx d$ away from the crack surface (dotted regions). (b) When two vertical cracks are so close that the region of reduced elastic deformation overlap, then the total reduction in the elastic energy is reduced which corresponds to a repulsive force acting between the cracks. As a result, at high concentration of cracks, they try to stay as far away from each other as possible, resulting in a hexagonal crack pattern.

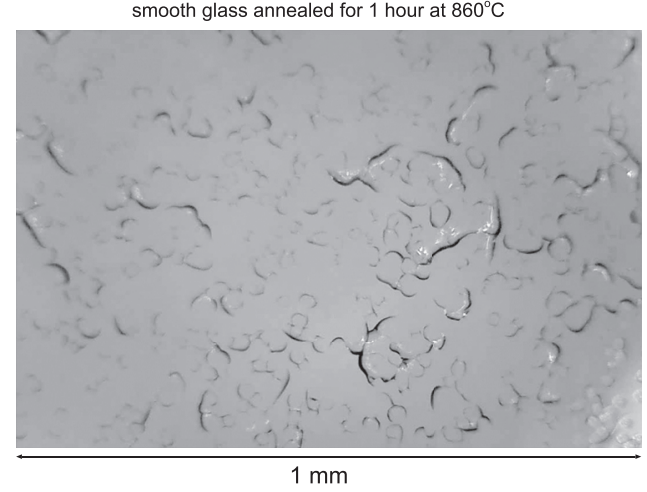

FIG. 16. Optical picture of the not sandblasted glass surface after annealing at $860^{\circ} \mathrm{C}$ for $1 \mathrm{~h}$. Note that the concentration of crack-like defects is smaller than on the sandblasted surface exposed to the same heat cycle (see Fig. 9). This may be due to a lower concentration of surface defects on the smooth glass plate as compared to that on the sandblasted glass plate, which can act as nucleus for the crack formation.

from the crack surface (see Fig. 15). Hence, if the temperature is lowered by $\Delta \mathrm{T}$ in a layer at the surface of thickness $d$, then the elastic energy stored in the surface layer will nearly vanish if a hexagonal pattern of cracks is formed, with the cell diameter $d$ and extending a distance $d$ into the solid. However, the hexagonal crack system can form only if the elastic energy of the un-cracked surface layer, which is of order $\mathrm{E}(\alpha \Delta \mathrm{T})^{2} \mathrm{~A}_{0} d$ (where $\mathrm{A}_{0}$ is the surface area, $\alpha$ is the thermal expansion coefficient, and E is Young's elastic modulus), is larger than the crack system surface energy, which is of order $\left(\mathrm{A}_{0} / d^{2}\right) \gamma d^{2}$ (where $\gamma$ is the surface energy per unit surface area). Thus we get $d>d_{\mathrm{c}}$, where $d_{\mathrm{c}}=(\gamma / \mathrm{E})(\alpha \Delta \mathrm{T})^{-2}$, as a necessary condition for the formation of the system of cracks. Assuming $\Delta \mathrm{T}$ of order a few $100{ }^{\circ} \mathrm{C}$ for silica glass, $d_{\mathrm{c}}=10-100 \mu \mathrm{m}$.

An accurate study of the formation of the crack pattern must take into account that with the increasing crack length the mutual unloading of cracks increases (leading to bifurcation-type instability $)^{30}$ and also that for a perfectly smooth glass surface no defects exist which can act as nucleus for the formation of cracks. ${ }^{27}$ For the sandblasted surface, the latter is not a problem, and this may explain why a more welldefined polygonal crack pattern (with a larger concentration of cracks) is formed on the sandblasted surface than on the not-sandblasted glass surface (see Fig. 16), when exposed to the same heat cycle.

\section{REFERENCES}

${ }^{1}$ B. N. J. Persson, Sliding Friction: Physical Principles and Applications (Springer, Heidelberg, 2000).

${ }^{2}$ E. Gnecco and E. Meyer, Elements of Friction Theory and Nanotribology (Cambridge University Press, 2015).

${ }^{3} \mathrm{~J}$. N. Israelachvili, Intermolecular and Surface Forces, 3rd ed. (Academic, London, 2011).

${ }^{4}$ J. R. Barber, Contact Mechanics (Solid Mechanics and Its Applications) (Springer, 2018). 
${ }^{5}$ B. N. J. Persson, "Contact mechanics for randomly rough surfaces," Surf. Sci. Rep. 61, 201 (2006).

${ }^{6}$ B. N. J. Persson, "Theory of rubber friction and contact mechanics," J. Chem. Phys. 115, 3840 (2001)

${ }^{7}$ M. H. Müser, W. B. Dapp, R. Bugnicourt, P. Sainsot, N. Lesaffre, T. A. Lubrecht, Bo N. J. Persson, K. Harris, A. Bennett, K. Schulze, S. Rohde, I. Peter, W. G. Sawyer, T. Angelini, H. A. Esfahani, M. Kadkhodaei, S. Akbarzadeh, J.-J. Wu, G. Vorlaufer, A. Vernes, S. Solhjoo, A. I. Vakis, R. L. Jackson, Y. Xu, J. Streator, A. Rostami, D. Dini, S. Medina, G. Carbone, F. Bottiglione, L. Afferrante, J. Monti, L. Pastewka, M. O. Robbins, and J. A. Greenwood, "Meeting the contact-mechanics challenge," Tribiol. Lett. 65, 118 (2017).

${ }^{8}$ A. I. Vakis, V. A. Yastrebov, J. Scheibert, C. Minfray, L. Nicola, D. Dini, A. Almqvist, M. Paggi, S. Lee, G. Limbert, J. F. Molinari, G. Anciaux, R. Aghababaei, S. E. Restrepo, A. Papangelo, A. Cammarata, P. Nicolini, C. Putignano, G. Carbone, M. Ciavarella, S. Stupkiewicz, J. Lengiewicz, G. Costagliola, F. Bosia, R. Guarino, N. M. Pugno, and M. H. Müser, "Modeling and simulation in tribology across scales: An overview," Tribol. Int. 125, 169 (2018).

${ }^{9}$ J. Krim, I. Heyvaert, C. Van Haesendonck, and Y. Bruynseraede, "Scanning tunneling microscopy observation of self-affine fractal roughness in ionbombarded film surfaces," Phys. Rev. Lett. 70, 57 (1993).

${ }^{10} \mathrm{~J}$. Krim and J. O. Indekeu, "Roughness exponents: A paradox resolved," Phys. Rev. E 48, 1576 (1993).

${ }^{11}$ H. Yan, "Kinetic growth with surface diffusion: The scaling aspects," Phys. Rev. Lett. 68, 3048 (1992).

${ }^{12}$ B. N. J. Persson, "On the fractal dimension of rough surfaces," Tribiol. Lett. 54, 99 (2014)

${ }^{13}$ I define a randomly rough surface as a surface which can be obtained by adding plane waves with random and uncorrelated phases $\phi_{\mathrm{q}}$, i.e., $h(\mathbf{x})$ $=\sum_{\mathbf{q}} \mathrm{B}_{\mathrm{q}} \exp \left(i \mathbf{q} \cdot \mathbf{x}+i \phi_{\mathrm{q}}\right)$. Such a surface has a Gaussian height probability distribution and hence zero skewness.

${ }^{14}$ B. N. J. Persson, O. Albohr, U. Tartaglino, A. I. Volokitin, and E. Tosatti, "On the nature of surface roughness with application to contact mechanics, sealing, rubber friction and adhesion," J. Phys.: Condens. Matter 17, R1 (2005).

${ }^{15}$ T. D. B. Jacobs, T. Junge, and L. Pastewka, "Quantitative characterization of surface topography using spectral analysis," Surf. Topogr.: Metrol. Prop. 5, 013001 (2017)
${ }^{16}$ Note that different authors use different prefactors in the definition of the power spectrum. Thus, for example, in Ref. 15 the 1D power spectrum is without the factor of $1 /(2 \pi)$ in the integral (1) so that $C_{1 D}^{*}=2 \pi C_{1 D}$, where $\mathrm{C}_{1 \mathrm{D}}^{*}$ is the $1 \mathrm{D}$ power spectrum used in Ref. 15 .

${ }^{17}$ See http: / / glassproperties.com/viscosity for information about the temperature dependency of the viscosity of borosilicate glass.

${ }^{18}$ N. M. Parikh, "Effect of atmosphere on surface tension of glass," J. Am. Ceram. Soc. 41, 18 (1958).

${ }^{19}$ B. Lorenz and B. N. J. Persson, "Leak rate of seals: Effective-medium theory and comparison with experiment," Eur. Phys. J. E 31, 159 (2010).

${ }^{20}$ P.-G. de Gennes, F. Brochard-Wyart, and D. Quere, Capillarity and Wetting Phenomena: Drops, Bubbles, Pearls, Waves (Springer, 2004).

${ }^{21}$ F. Bottiglione, G. Carbone, and B. N. J. Persson, "Fluid contact angle on solid surfaces: Role of multiscale surface roughness," J. Chem. Phys. 143, 134705 (2015).

${ }^{22}$ A. L. Sumner, E. J. Menke, Y. Dubowski, J. T. Newberg, R. M. Penner, J. C. Hemminger, L. M. Wingen, T. Brauersc, and B. J. Finlayson-Pitts, "The nature of water on surfaces of laboratory systems and implications for heterogeneous chemistry in the troposphere," Phys. Chem. Chem. Phys. 6, 604 (2004).

${ }^{23}$ L. Landau and E. Lifshitz, Fluid Mechanics, 3rd ed. (Pergamon, Oxford, 1966).

${ }^{24}$ V. Levich, Physicochemical Hydrodynamics (Prentice Hall, Englewood Cliffs, NJ, 1962).

${ }^{25}$ Z. P. Bazant and L. Cedolin, Stability of Structures: Elastic, Inelastic Fracture and Damage Theories (Oxford University Press, New York, 1991).

${ }^{26}$ M. Hofmann, R. Anderssohn, H. A. Bahr, H. J. Weiß, and J. Nellesen, "Why hexagonal basalt columns?," Phys. Rev. Lett. 115, 154301 (2015).

${ }^{27}$ B. Bourdin, J. J. Marigo, C. Maurini, and P. Sicsic, "Morphogenesis and propagation of complex cracks induced by thermal shocks," Phys. Rev. Lett. 112, 014301 (2014).

${ }^{28}$ E. A. Jagla and A. G. Rojo, "Sequential fragmentation: The origin of columnar quasihexagonal patterns," Phys. Rev. E 65, 026203 (2002).

${ }^{29}$ E. A. Jagla, "Maturation of crack patterns," Phys. Rev. E 69, 056212 (2004).

${ }^{30}$ H. A. Bahr, H. J. Weiss, U. Bahr, M. Hofmann, G. Fischer, S. Lampenscherf, and H. Balke, "Scaling behavior of thermal shock crack patterns and tunneling cracks driven by cooling or drying," J. Mech. Phys. Solids 58, 1411 (2010). 